OPEN ACCESS

Edited by:

Dörthe Schaue,

UCLA David Geffen School of

Medicine, United States

Reviewed by:

Tomer Charas,

Rambam Health Care Campus, Israel

Bilgin Kadri Aribas,

Bülent Ecevit University, Turkey

*Correspondence:

Weihua Liao

owenliao@csu.edu.cn

You-Ming Zhang

zhangym0820@csu.edu.cn

Specialty section:

This article was submitted to

Radiation Oncology,

a section of the journal

Frontiers in Oncology

Received: 29 March 2021

Accepted: 14 June 2021

Published: 12 July 2021

Citation:

Zhao L-M, Kang Y-F, Gao J-M,

Li L, Chen R-T, Zeng J-J,

Zhang Y-M and Liao W-H (2021)

Functional Connectivity Density for

Radiation Encephalopathy Prediction

in Nasopharyngeal Carcinoma.

Front. Oncol. 11:687127.

doi: 10.3389/fonc.2021.687127

\section{Functional Connectivity Density for Radiation Encephalopathy Prediction in Nasopharyngeal Carcinoma}

\author{
Lin-Mei Zhao ${ }^{1}$, Ya-Fei Kang ${ }^{2}$, Jian-Ming Gao ${ }^{3}$, Li Li ${ }^{4}$, Rui-Ting Chen ${ }^{1}$, Jun-Jie Zeng ${ }^{5}$, \\ You-Ming Zhang ${ }^{1 *}$ and Weihua Liao ${ }^{1 *}$ \\ ${ }^{1}$ Department of Radiology, Xiangya Hospital, Central South University, Changsha, China, 2 School of Psychology, Shaanxi \\ Normal University, Shanxi Provincial Key Research Center of Child Mental and Behavioral Health, Xi'an, China, ${ }^{3}$ Department \\ of Radiation Oncology, State Key Laboratory of Oncology in South China, Collaborative Innovation Center for Cancer \\ Medicine, Sun Yat-sen University Cancer Center, Guangzhou, China, ${ }^{4}$ State Key Laboratory of Oncology in South China, \\ Collaborative Innovation Center for Cancer Medicine, Sun Yat-sen University Cancer Center, Guangzhou, China, \\ ${ }^{5}$ Department of Radiology, Hunan Children's Hospital, Changsha, China
}

The diagnostic efficiency of radiation encephalopathy (RE) remains heterogeneous, and prediction of RE is difficult at the pre-symptomatic stage. We aimed to analyze the wholebrain resting-state functional connectivity density (FCD) of individuals with presymptomatic RE using multivariate pattern analysis (MVPA) and explore its prediction efficiency. Resting data from NPC patients with nasopharyngeal carcinoma (NPC; consisting of 20 pre-symptomatic RE subjects and 26 non-RE controls) were collected in this study. We used MVPA to classify pre-symptomatic RE subjects from non-RE controls based on FCD maps. Classifier performances were evaluated by accuracy, sensitivity, specificity, and area under the characteristic operator curve. Permutation tests and leave-one-out cross-validation were applied for assessing classifier performance. MVPA was able to differentiate pre-symptomatic RE subjects from non-RE controls using global FCD as a feature, with a total accuracy of $89.13 \%$. The temporal lobe as well as regions involved in the visual processing system, the somatosensory system, and the default mode network (DMN) revealed robust discrimination during classification. Our findings suggest a good classification efficiency of global FCD for the individual prediction of RE at a pre-symptomatic stage. Moreover, the discriminating regions may contribute to the underlying mechanisms of sensory and cognitive disturbances in RE.

Keywords: radiotherapy, nasopharyngeal carcinoma, magnetic resonance imaging, machine learning, follow-up 


\section{INTRODUCTION}

Nasopharyngeal carcinoma (NPC) is a malignancy stemming from the nasopharyngeal epithelium, and more than $70 \%$ of all new cases are confirmed in the east and southeast Asia (1). Recently, the optimization of radiotherapy and chemotherapy strategies has considerably improved disease control and survival (2). Nevertheless, some long-term treatment-related complications still seriously affected the patients' quality of life. This is especially true of radiation encephalopathy (RE), which has captured the attentions of clinicians and researchers alike for its deteriorating neuropsychiatric symptoms, sometimes even causing death (3). Early intervention has been reported to improve patient prognosis; however, existing conventional magnetic resonance imaging (MRI) techniques can only discern RE at the irreversible stage (4). The early identification or individualized prediction of RE is therefore crucial for improving quality of life and prognosis in patients with RE.

The advent of other neuroimaging techniques has enabled the earlier detection of radiation-induced alterations in patients with NPC (5-7). The neuroimaging index reflects a disease-specific pathological or neurophysiological property and may even be an early biomarker of such alterations. For morphology, one gray matter morphology-based study has suggested that cortical surface area might be a morphological marker of patients with early-stage RE (8). In addition, a white matter connectivity-based structural network study revealed a network-level reorganization in the late-delayed stages of RE (9). However, most studies have mainly focused on the differences at group levels; far less attention has been paid to the potential value of individual levels.

With the emergence of multivariate pattern analysis (MPVA), the individual recognition of neurological diseases is possible. Several recent reports about the individualized prediction of RE have been promising. For example, a machine-learning study used texture features to develop radiomics models for the dynamic prediction of RE (10). However, these texture features were from the medial temporal lobe, and information from outside the medial temporal lobe was insufficiently investigated. Another recent support vector machine (SVM) study based on white matter integrity reported good abilities for diagnoses in different periods of RE (11). Unfortunately, the above discriminative power of gray matter-derived features has been largely overlooked.

A recent study has demonstrated that functional parameters are altered earlier and are more vulnerable than those that reflect structural integrity (5), suggesting that aberrance in functional domains may play a critical role in the pathogenesis of RE. Furthermore, using resting-state functional MRI (rs-fMRI), the

Abbreviations: 2DCRT, two-dimensional radiation therapy; ACC, anterior cingulum cortex; DPABI, Data Processing \& Analysis for Brain Imaging; DMN, default mode network; fALFF, fractional amplitude of low-frequency fluctuations; g/IFCD, global/local functional connectivity density; IMRT, intensity-modulated radiation therapy; MPVA, multivariate pattern analysis; NPC, nasopharyngeal carcinoma; PRoNTo, Pattern Recognition for Neuroimaging Toolbox; RE, radiation encephalopathy; ReHo, regional homogeneity; ROC, receiver operating characteristic; rs-fMRI, resting-state functional MRI; SBM, surfacebased morphometry; STG, superior temporal gyrus; SVM, support vector machine; VBM, voxel-based morphology. fact that neurophysiological characteristics of neuroimaging function alterations in RE involved the whole brain (12) makes large-scale functional evaluation notable. Functional connectivity density (FCD) allows researchers to evaluate the whole-brain functional brain connectivity patterns at the voxel level (13). It can reflect the early patterns of disease-specific neuronal activity changes (14-16). To the best of our knowledge, FCD has not yet been used to predict RE at the pre-symptomatic stage. Therefore, the combination of FCD and machine learning strategies in the present study may contribute to a better understanding of the pathological mechanisms of RE and aid in its early prediction.

\section{MATERIALS AND METHODS}

\section{Study Design and Subjects Enrollment}

We developed the MVPA from a cohort of 46 NPC patients. All participants were right-handed and had pathologically confirmed NPC. Other specific inclusion criteria were as follows: 1) aged between 20 and 60 years with over 6 years of education; 2) NPC patients who underwent radiotherapy within the previous 6 months; 3) no abnormalities of RE; and 4) no presentation of any other intracranial or central nervous system diseases. Patients were excluded if they had a consciousness disorder, central nervous system disease, or any other disease. All patients were treated with radiotherapy before the study using either two-dimensional radiation therapy (2DCRT) or intensity-modulated radiation therapy (IMRT). To control the confounding effect of chemotherapy on the FCD changes, all the enrolled patients treated with chemotherapy had balanced between group clinical stages, chemotherapy mode, regimens and chemotherapy type by reading their MR images and medical records (Table 1) (8). The detailed information of chemotherapy (such as chemotherapy agents, dose for each agent, time for medication administration, number of courses, and duration) for patients with NPC in this study could be obtained in Supplementary Materials (Table S1). The NPC patients were then divided into subgroups based on whether or not their conventional images meet the RE diagnoses criterion (3) during the follow-up (72 \pm 8 months). Specifically, the subsequent neuroimaging analysis was based on original data rather than followed-up data. The exact procedures are shown in the overall flowchart in Figure 1. Informed consent was obtained from all subjects, and the ethics committee approved the study before its execution.

\section{MRI Acquisition}

MRI images were collected using a 3.0-T MRI scanner (MAGNETOM Tim Trio, Siemens, Germany). Functional imaging data were generated from echo-planar imaging sequences, and the main parameters were as follows: repetition time $=2,400 \mathrm{~ms}$, echo time $=30 \mathrm{~ms}$, matrix size $=64 \times 64$, flip angle $=90^{\circ}$, number of timepoints $=240$, field of view $=230 \mathrm{~mm} \times$ $230 \mathrm{~mm}$, and 40 axial slices. During the rs-fMRI sessions, participants were asked to keep their eyes closed, without falling asleep or thinking of anything. Three-dimensional T1-weighted magnetization-prepared rapid acquisition with gradient echo sequences were taken as follows: 176 sagittal slices overall, voxel 
TABLE 1 | Demographic and clinical characteristics.

\begin{tabular}{|c|c|c|c|}
\hline Characteristics & $\begin{array}{l}\text { NPC patients } \\
\text { followed-up } \\
\text { with RE }(n=20)\end{array}$ & $\begin{array}{l}\text { NPC patients } \\
\text { followed-up } \\
\text { without RE } \\
\text { (n = 26) }\end{array}$ & $P$-value \\
\hline Age (year) & $45.10 \pm 9.63$ & $44.54 \pm 11.24$ & 0.86 \\
\hline Gender (male/female) & $15 / 5$ & $17 / 9$ & 0.53 \\
\hline Clincial stage & & & 0.883 \\
\hline $\mathrm{I} / \mathrm{II}, \mathrm{n}$ & $5(10.87 \%)$ & $7(15.22 \%)$ & \\
\hline III/IV, n & $15(32.61 \%)$ & $19(41.30 \%)$ & \\
\hline Teatment option & & & 0.289 \\
\hline Radiotherapy only, n & $2(4.35 \%)$ & $7(15.22 \%)$ & \\
\hline Radio-chemotherapy, n & $18(39.13 \%)$ & $19(41.30 \%)$ & \\
\hline Radiotherapy time (month) & $22.70 \pm 28.43$ & $32.54 \pm 27.43$ & 0.89 \\
\hline $\begin{array}{l}\text { Chemotherapy mode for } \\
\text { patients treated with radio- }\end{array}$ & & & \\
\hline $\begin{array}{l}\text { chemotherapy } \\
\text { Neoadjuvant and concomitant } \\
\text { chemotherapy, } \mathrm{n}\end{array}$ & $16(43.24 \%)$ & $18(48.65 \%)$ & 0.604 \\
\hline Others, $\mathrm{n}$ & $2(5.41 \%)$ & $1(2.70 \%)$ & \\
\hline $\begin{array}{l}\text { Chemotherapy regimens for } \\
\text { patients treated with radio- }\end{array}$ & & & \\
\hline chemotherapy & & & 0.447 \\
\hline TPF/TP/PF, n & $13(35.14 \%)$ & $16(43.24 \%)$ & \\
\hline $\mathrm{GP}, \mathrm{n}$ & $5(13.51 \%)$ & $3(8.11 \%)$ & \\
\hline Chemotherapy type & & & NA \\
\hline Target-directed chemotherapy, n & $0(0)$ & $0(0)$ & \\
\hline Conventional chemotherapy, $\mathrm{n}$ & $18(48.65 \%)$ & $19(51.35 \%)$ & \\
\hline
\end{tabular}

Note: NPC, nasopharyngeal carcinoma; RE, radiation encephalopathy; TPF, docetaxel, cisplatin and fluorouracil; TP, docetaxel and cisplatin; PF, cisplatin and fluorouracil; GP, gemcitabine and cisplatin; NA, not available. Clinical stage were obtained according to the 7th edition of the UICC/AJCC (2009) TNM. Stage I: T1NOMO; Stage II: TO-1N1MO and T2NO-1MO; Stage III: TO-2N2MO and T3NO-2MO; Stage IV: T4NO-2MO, or N3 or M1.

size $=1.0 \mathrm{~mm} \times 1.0 \mathrm{~mm} \times 1.0 \mathrm{~mm}$, slice thickness $/$ gap $=1.0 / 0 \mathrm{~mm}$, matrix size $=256 \times 256$, field of view $=256 \mathrm{~mm} \times 256 \mathrm{~mm}$, repetition time $=2,300 \mathrm{~ms}$, echo time $=2.98 \mathrm{~ms}$, flip angle $=9^{\circ}$. Routine sequences were scanned to ensure a diagnosis of RE and exclude other diseases.

\section{FCD Analysis}

The rs-fMRI data were first preprocessed using the Data Processing \& Analysis for Brain Imaging (DPABI) toolbox (https://rfmri.org/dpabi) (17), and the initial 10 volumes were removed. Next, slice-timing, realignment, spatial normalization, regression of nuisance covariance, and temporal filtering steps were performed. The preprocessed data were then used for FCD mapping calculation with an in-house script in MATLAB according to the methods described by Tomasi and Volkow (13). FCD mapping was used to compute the global FCD (gFCD) as well as the local FCD (IFCD) in identified distributions of hubs in the brain (18). Further details are documented below.

\section{Data Preprocessing}

A toolbox for Data Processing \& Analysis for Brain Imaging (19) (DPABI; https://rfmri.org/dpabi) pipeline was used to preprocess the rs-fMRI data, as follows: (1) The first 10 volumes were removed to adapt subjects to the scanning environment and lower the magnetization equilibrium; (2) Slice-timing correction: the proper slice order and reference order were selected; (3) Realignment: the time series of each subject were realigned using a linear transformation with six-parameter (rigid-body) and head motion correction [translational displacement $[\mathrm{x}, \mathrm{y}$, or $\mathrm{z}$ directions] $<2.0 \mathrm{~mm}$, or maximum rotation $\left.<2.0^{\circ}(20)\right]$ were carried out; individual three-dimensional T1 images were subsequently co-registered to mean resting images using linear transformations ( $6^{\circ}$ of freedom) without re-sampling and were later segmented into the different components of gray matter, white matter and cerebrospinal fluid; (4) Spatial normalization was performed using the DARTEL tool (21) for transformations from the individual native space to the MNI space $(3 \mathrm{~mm} \times$ $3 \mathrm{~mm} \times 3 \mathrm{~mm}$ voxel size); (5) Linear regression was performed for nuisance covariates, including head motor parameters from the realignment step (the Friston 24-parameter model), global mean signals, white matter, and cerebrospinal fluid signals; and (6) All available images were temporally filtered with a $0.01-0.08$ $\mathrm{Hz}$ bandpass to minimize the effects of high-frequency physiological noises and low-frequency drift.

\section{FCD Calculation}

The FCD calculation was restricted to voxels within the gray matter mask, which was predefined through tissue with probabilities of more than $20 \%$ in the gray matter probability template (22). Pearson correlation coefficient at the threshold of $\mathrm{R}>0.6$ determined the functional connectivity between voxels. We selected this threshold of 0.6 because of its relatively high specificity and sensitivity (14). The related scripts were showed in Supplementary Material.

\section{IFCD}

The lFCD of a given voxel (x0) was computed using a "growing" algorithm. Specifically, the number of functional connections for any given voxel (xn) and its adjacent voxels (xni) was calculated. First, the time series of a given voxel $(\mathrm{x} 0)$ and its adjacent voxels (xi) were calculated using Pearson correlation analysis. Each xi was added to a cluster only when the Pearson correlation coefficient was larger than the threshold ( Ri0 >0.6). Next, the Pearson correlation for a time-varying series between $\mathrm{x} 0$ and a voxel (xj) adjacent to xi was also evaluated; similarly, each xj was added to the aforementioned cluster when $\mathrm{Rj} 0>0.6$. This process was repeated in an iterative way for all other voxels $(\mathrm{N}-1)$ that were adjacent to voxels in the aforementioned cluster and functionally connected to $\mathrm{x} 0$, until no fresh voxels were able to be added to the cluster. The IFCD at $\mathrm{x} 0$ was defined as the number of units in the local functional connectivity cluster, $\mathrm{k}$ $(\mathrm{x} 0)$. After finishing this process for a given voxel $(\mathrm{x} 0)$, the calculation was initiated for a different given voxel. This calculation was performed for all $\mathrm{N}$ voxels.

\section{gFCD}

The gFCD for a given voxel $\mathrm{x} 0$ was defined as the global number of functional connections, $\mathrm{k}(\mathrm{x} 0)$, between $\mathrm{x} 0$ and all other global voxels. This calculation was also iterated for all given voxels $(\mathrm{N})$ in the global brain and underwent the operation of $\mathrm{N} \times(\mathrm{N}-1) /$ 2 correlations.

All FCD maps were normalized to the average FCD of individual whole brains (FCD normalized $[\mathrm{x}, \mathrm{y}, \mathrm{z}]=\mathrm{FCD}[\mathrm{x}, \mathrm{y}, \mathrm{z}]$ /mean FCD [k0]). Finally, all FCD maps were spatially smoothed 


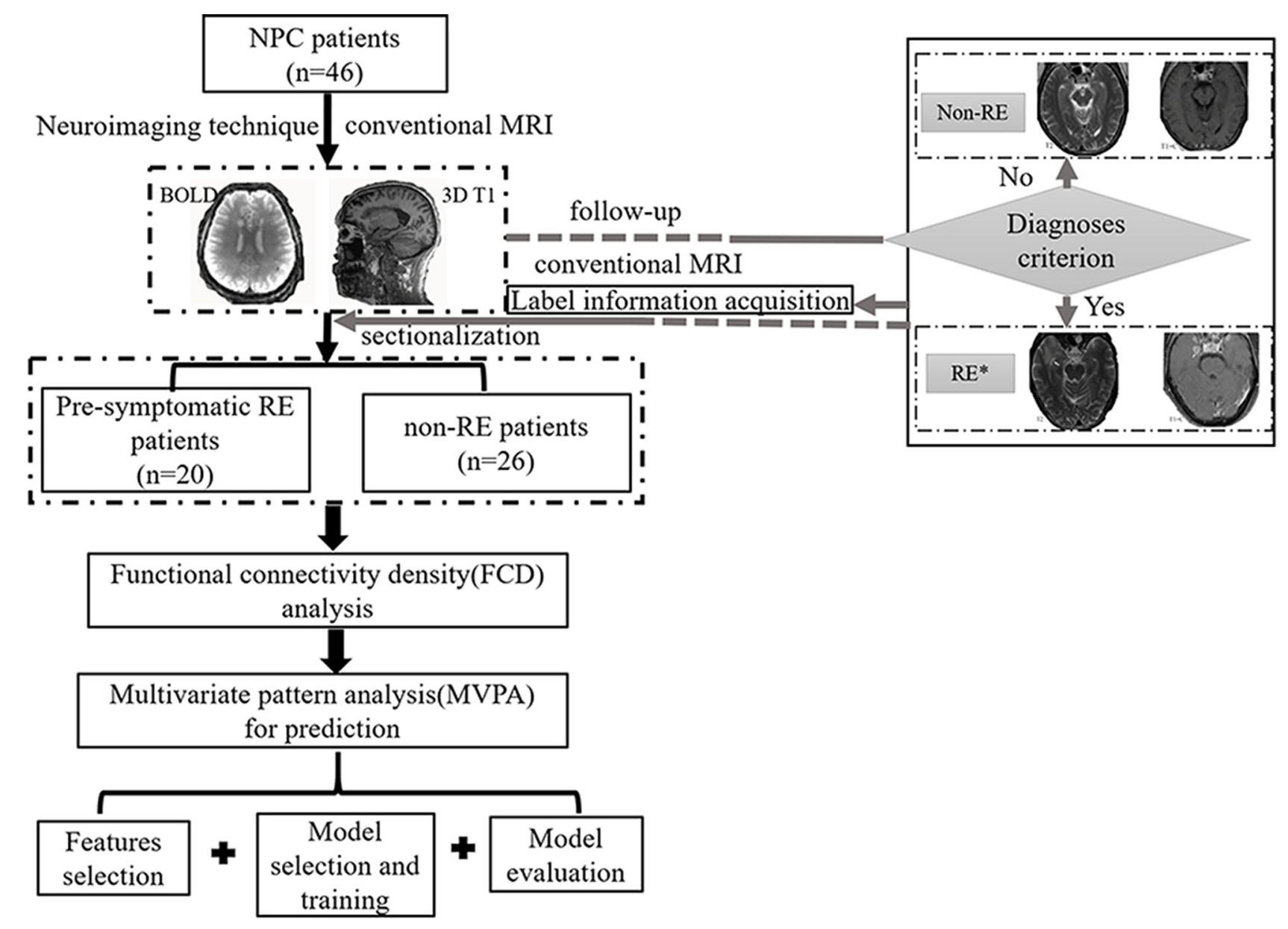

FIGURE 1 | The flowchart of this study. ${ }^{*}$, patients with radiation encephalopathy were confirmed by Merritt's Neurology; NPC, nasopharyngeal carcinoma; RE, radiation encephalopathy; BOLD, blood oxygen level-dependent; T2, T2 weighted image; T1+C, T1 weighted image + contrast.

using an $8 \mathrm{~mm}$ full-width at half-maximum (FWHM) Gaussian kernel before the subsequent analysis.

\section{Statistical Analysis}

Demographic information and FCD maps were compared between pre-symptomatic RE and non-RE groups. Unpaired ttests and $\chi 2$ tests were used to analyze demographic information. Unpaired t-tests were conducted to compare FCD maps with age, gender, and years of education as covariates. $\mathrm{P}<0.05$ was considered to indicate statistical significance.

\section{SVM Analysis Using FCD}

A linear kernel SVM algorithm was applied based on Pattern Recognition for Neuroimaging Toolbox (PRoNTo version 2.0, http://www.mlnl.cs.ucl.ac.uk/pronto) to estimate the underlying brain regions that most contributed to classifying presymptomatic RE versus non-RE subjects (23). The central bodies of the SVM method were briefly concluded as follows: 1) features extraction and selection, 2) discriminative regions selection, 3) the SVM classifier model training using the training data, and 4) evaluation of the performances of the SVM model using the evaluation data.

In this present study, feature selection consisted of the FCD values that were expected to show statistical significance between the two groups. The procedures aforementioned above were automatically processed using Prepare feature set pipeline of PRoNTo.

The leave-one-out cross-validation method was applied to validate the SVM classifier's validation. Each time, feature selection was conducted using the training data to avoid circularity effects. The training data in this step involved ( $\mathrm{n}-$ 1) subjects, and the excluded single subject was used to test the generalization ability (i.e., the ability to reliably classify new samples). These above steps were repeated $n$ times $(n=$ the number of subjects) until the classifier generalizability was unbiased. The process was automatically computed using the 'specify model' pipeline of PRoNTo.

Classifier performance was evaluated by its accuracy, sensitivity, specificity, and area under the receiver operating characteristic operator curve (AUC), with the procedure 
repeated for each pair of the subject. Furthermore, a 5,000 times non-parametric permutation test performed the evaluation, with corrected $\mathrm{P}<0.05$ denoting significance in this evaluation. The aforementioned procedures were selected and automatically computed using the 'run model' and 'display results' pipelines of PRoNTo.

The 'compute weights' and 'display weights' pipelines were also run using PRoNTo. These pipelines produced the voxel weight vectors and a list of regions in descending order according to their contributions to the classification model. The voxel weight vectors were subsequently converted to a map, which was visualized using the BrainNet Viewer (24).

\section{RESULTS}

\section{Demographic and Clinical Characteristics}

The demographic and related clinical results are displayed in Table 1. The two groups were matched for age, gender, clinical stage, treatment options, and therapy time. Chemotherapy parameters such as chemotherapy mode, regimens, and types were not significantly different between the two groups $(P>0.05)$.

\section{Classification Results}

The gFCD was significantly different between the two groups $(P<0.05)$, while the IFCD was not. We therefore selected the $\mathrm{gFCD}$ as the feature for classification. The linear SVM analysis predicted a diagnosis of RE using gFCD with a total accuracy of $89.13 \%$ and a balanced accuracy of $88.08 \%$ (sensitivity of $80.00 \%$, and a specificity of $96.15 \%$ ). The receiver operating characteristic (ROC) curve and AUC were also plotted (Figure 2). The AUC was 0.97, and permutation tests for the AUC revealed statistical significance.

\section{Brain Weighted Location Model}

For the gFCD, we presented the weighted voxel distribution to classify between RE patients and non-RE patients (Figure 3). The top 20 spatial distribution in terms of normalized weights per region was revealed to $43.29 \%$ of the predictive weights (Table S2). These regions identified (Figure 4) through weighted landmarks mainly included the bilateral temporal pole and cuneus. Unilateral regions of the left hemisphere covered the superior temporal cortex, middle occipital cortex, amygdala, angular and supramarginal cortex, and anterior cingulum cortex (ACC). In contrast, regions of the right hemisphere consisted of the opercular and triangular parts of the inferior frontal cortex, the parahippocampus, and the postcentral and precuneus gyri, together with part of the right regions of the cerebellum and its crus.

\section{DISCUSSION}

This was the first study to examine FCD alterations between presymptomatic RE and non-RE NPC patients, which were then used to predict the occurrence of RE using a machine learning approach. The FCD analysis revealed that gFCD was altered in

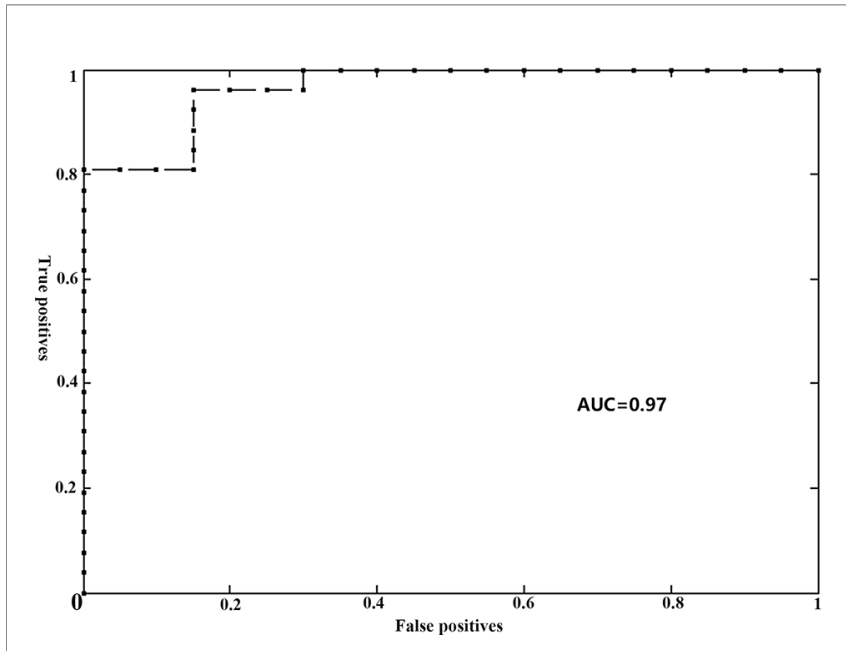

FIGURE 2 | Receiver operator curve (ROC) for individual prediction of RE at the pre-symptomatic stage in patients with NPC. RE, radiation encephalopathy; NPC, nasopharyngeal carcinoma; AUC, the area under the curve.

patients with pre-symptomatic RE. Upon closer inspection of these results, we revealed that brain regions with gFCD abnormalities were mainly found in the bilateral temporal lobe, as well as in regions involved in the visual pathway, the somatosensory system, and the default mode network (DMN). Moreover, gFCD alterations in these brain regions were able to predict RE in high performance with an accuracy of $89.13 \%$. This finding suggests that gFCD may be a novel imaging biomarker for the early detection of RE, which may contribute to a better understanding of its pathogenesis.

\section{Classification Interpretation}

In this current study, the predictive performance of the model was generally favorable, as evidenced by its accuracy of $89.13 \%$ and an AUC of 0.97. The prediction efficiency observed in our study was also higher than those of previous MVPA studies (10, $11,25)$. For example, using functional connectivity as a feature, $\mathrm{Ma}$ et al. reported an accuracy of $81.36 \%$ for differentiating patients with and without RE (25). Another MVPA study used fractional anisotropy or white matter connections to identify the individuals at a high risk of RE, with a maximum accuracy of 84.5\% (11). Furthermore, based on conventional MRI, a recent radiomics model study reported a maximum AUC of 0.83 for predicting RE (10). We speculated that different feature selection and/or modeling strategies might be responsible for the inconsistencies in the prediction accuracies for RE among these studies. Given that distinct features may reflect a specific physiological process, our findings of a better performance in the prediction of RE using FCD suggested that FCD may be a sensitive neuroimaging biomarker for reflecting the radiationinduced functional impairments.

\section{Brain Weight Location Model}

Notably, we found that gFCD, rather than IFCD, made a substantial contribution to the predictive model for the early 


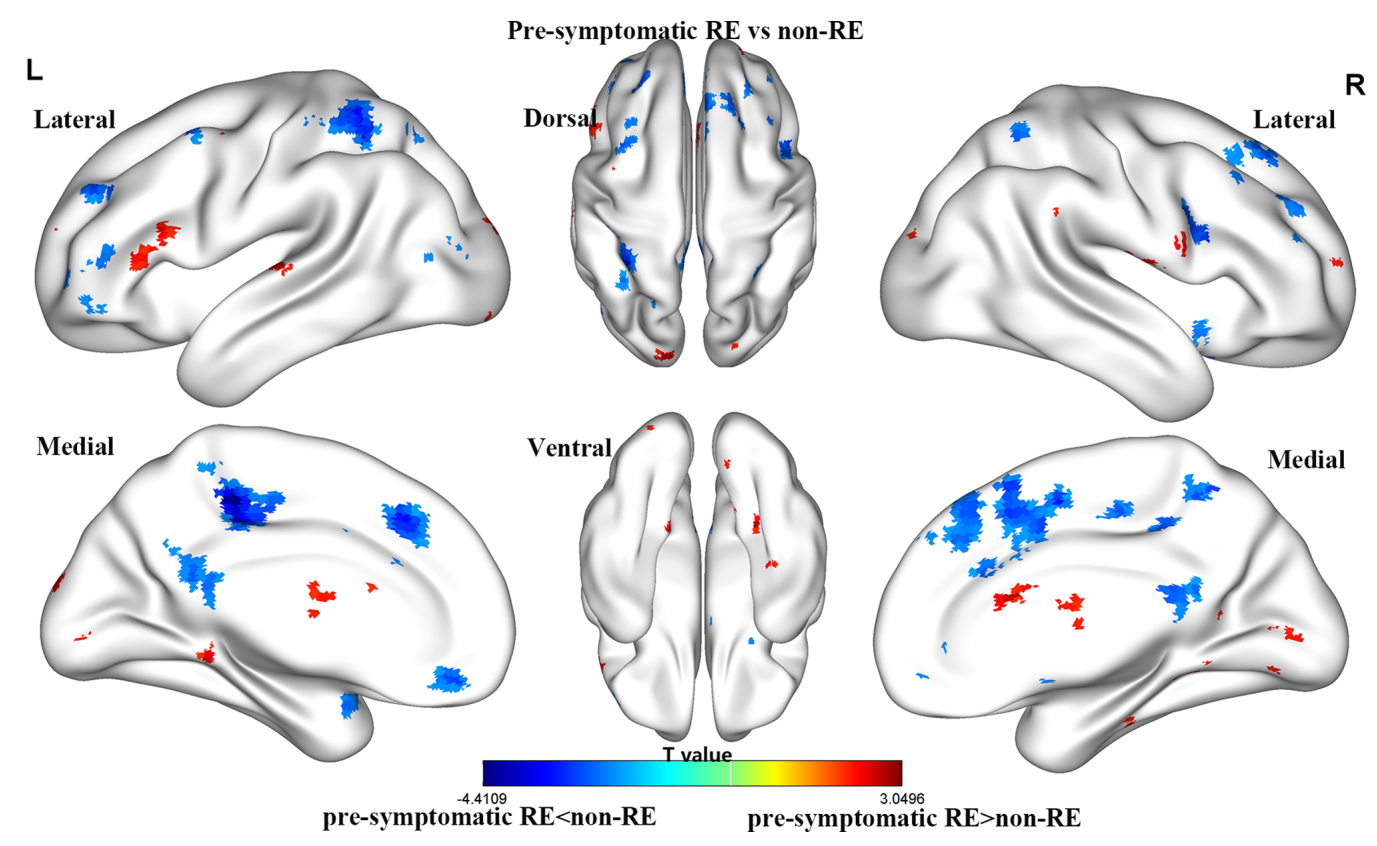

FIGURE 3 | The brain maps of pre-symptomatic RE and non-RE based on gFCD at the voxel level. RE, radiation encephalopathy; NPC, nasopharyngeal carcinoma; FCD, functional connectivity density.

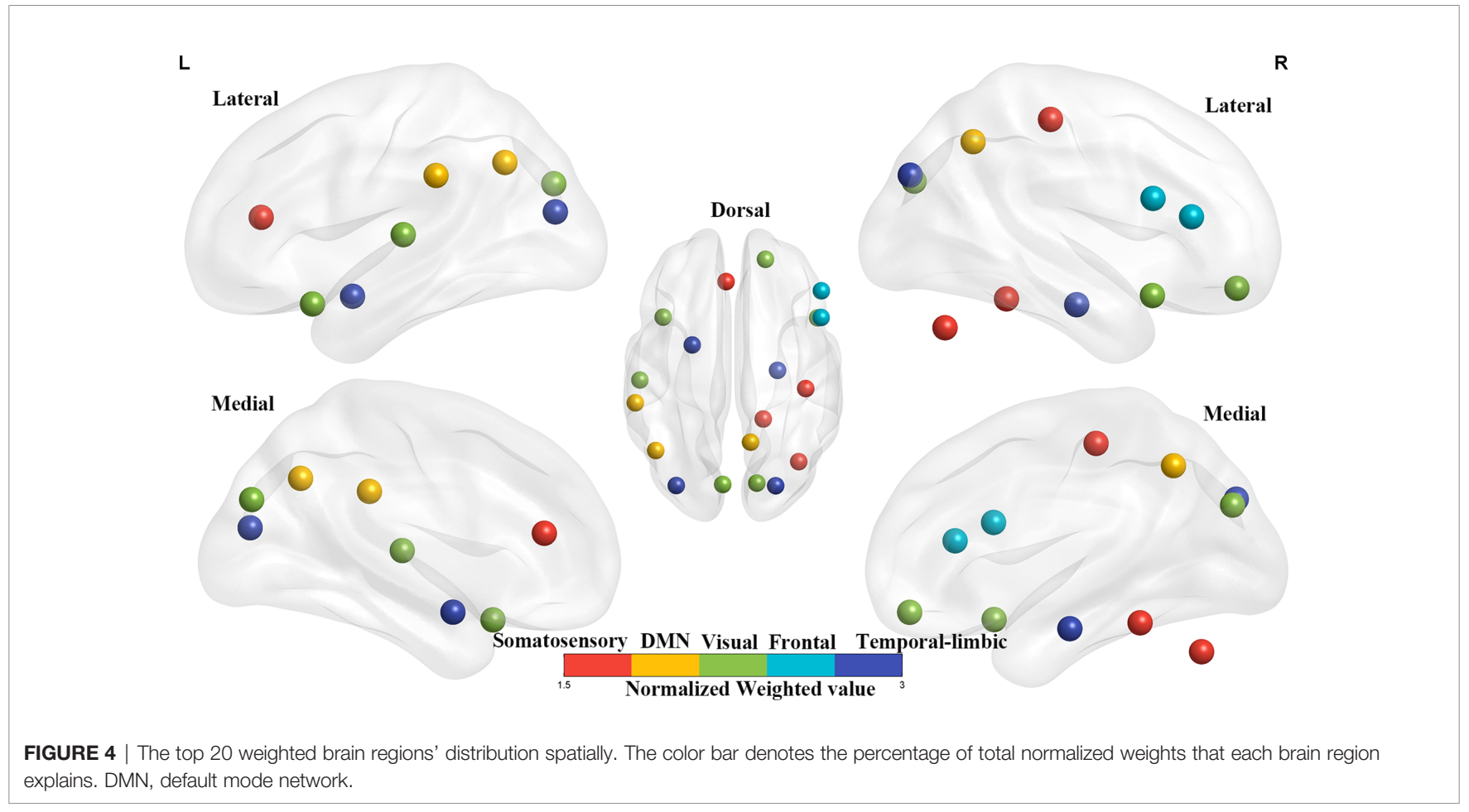

diagnosis of RE. Our results are partially supported by those of previous studies, which demonstrated that gFCD is more sensitive to individual differences than IFCD in terms of functional connectivity (18). It has been well documented that altered
gFCD is linked to functional deficits in multiple domains [such as attention (15), cognition (26), memory, and visual perceptual (16)], which are all common clinical symptoms in patients with RE $(3,27)$. Although the potential factors secondary to pathological 
alterations of radiation-induced vascular endothelial cell injury and vascular stenosis may lead to FCD abnormalities (28), the exact neural mechanisms underlying the observed patterns of FCD changes remain unclear.

The current study revealed that gFCD in the temporal regions and cuneus had good identification efficiency in patients with RE. These results were not surprising; the temporal areas [including the medial and inferior aspects (29)] are located in the radiation field and are thus vulnerable to injury (30). Recently, several neuroimaging studies $(7,8)$ have reported structural alterations in the temporal lobe following radiation therapy (31). For example, using voxel-based morphology (VBM) (31), decreased cortical volumes of the temporal regions were reported after radiation therapy in patients with NPC. One surface-based morphometry study reported increased cortical thickness of the left superior temporal gyrus (STG) in patients with NPC following radiation therapy (7). Another SBM study (32) revealed an increased cortical surface area in the temporal lobe and decreased cortical thickness in the bilateral temporal pole and STG. Aside from the structural evidence, our findings of altered gFCD in the temporal lobe are further supported by previously documented radiation-induced functional impairments (such as abnormal regional homogeneity ( $\mathrm{ReHo}$ ) and functional connectivity) in the temporal pole and STG $(5,12)$. Of note, the temporal gyrus and cuneus, where gFCD was altered in our study, can integrate visual information from the anterior visual pathway (33), whose lower stream (eyes lens, optic nerve, and optic chiasm) undergoes severe radiationinduced damage (34). We therefore speculated that the altered gFCD in the cuneus and temporal regions might reflect functional impairments in the anterior visual processing pathway (cuneus-temporal lobe loop). Furthermore, a previously reported increase in visual evoked potential latency and a decrease in the amplitude (35) of the anterior visual pathway of patients with RE may further support our hypothesis.

In our study, gFCD in the postcentral gyrus and ACC also contributed substantially to the early diagnosis of RE. Our findings are partially supported by several previous functional studies $(6,12)$, which reported increased ReHo in the postcentral gyrus and decreased FC in the postcentral gyrus and ACC. Furthermore, one SBM study provided structural evidence with cortical thickness abnormalities in the postcentral gyrus and ACC in patients with NPC after radiation therapy (7). Physiologically, the ACC receives inputs from the spinothalamic tract (36), which then projects to the postcentral gyrus, thus constituting the somatosensory pathway. One case report has also demonstrated that the injury of the spinothalamic tract can occur as a result of the primary brainstem injury (37), as the brainstem is located in the radiation field and receives a high radiation dose in patients with NPC (34). Taken together, the abnormal brain activity of the postcentral gyrus and ACC thus be a secondary response to the damaged sensory neural circuit in the brainstem. Moreover, sensory deficits, such as facial (38) and limb numbness or pain perception (39), that are observed in NPC patients after radiotherapy suggest that functional impairments occur in the sensory in the neural circuit.
We observed that the gFCD within the precuneus, supramarginal gyrus, and angular gyrus was crucial for predicting RE. The precuneus (32), and the inferior parietal cortex (supramarginal gyrus and angular gyri), are functionally connected and formed a resting-state brain network, known as the DMN. As has been reported, the DMN has self-referential, introspective-state functions, and processes an individual's thoughts and feelings $(40,41)$. To date, many previous studies have identified structural and functional abnormalities in DMNassociated brain regions, such as decreased cortical thickness (7), the abnormal fractional amplitude of low-frequency fluctuations (fALFF) (5), ReHo, and FC $(6,12)$. Thus, together with the previous observations, our results indicated that the activity of DMN activity might be a potential neurological biomarker for radiation-induced cognitive impairments; however, this needs further investigation.

\section{Limitations}

Some limitations were presented in this study. First, the study contained a relatively small series of patients because of the relatively low morbidity of RE as well as low patient compliance during follow-up. Although the current SVM algorithm was appropriate for a small sample size, future studies would benefit from a larger sample and would have a more stable predictive performance. We have thus started to create a larger $\mathrm{RE}$ database for further investigations. Secondly, the lack of any comprehensive assessments of cognitive function weakens the interpretability of our results. Future studies that use detailed cognitive scales will be indispensable for the validation of such an investigation. Thirdly, chemotherapy has been reported to exert side effects on the cerebral functional domain in patients with NPC following radiotherapy. We tried to control for the effects of confounding factors by keeping TNM stages and chemotherapy regimens consistent. However, further research is warranted to exclude the chemotherapy-related confounding effects on the radiation-induced functional impairments.

\section{CONCLUSIONS}

In the current study, we analyzed FCD maps using a machine learning SVM algorithm to predict RE in NPC patients for the first time. The gFCD was revealed to have a good prediction efficiency. This finding provides insights into voxel-level cerebral information and suggests that gFCD might be a valid biomarker of RE. Furthermore, brain regions within the temporal pole and those involved in visual processing, the somatosensory system, and the DMN showed high discrimination, which may help to explain the sensory and cognitive disturbances that occur in RE.

\section{DATA AVAILABILITY STATEMENT}

The original contributions presented in the study are included in the article/Supplementary Material. Further inquiries can be directed to the corresponding authors. 


\section{ETHICS STATEMENT}

The studies involving human participants were reviewed and approved by the Ethics Committee of Xiangya Hospital, Central South University. The patients/participants provided their written informed consent to participate in this study.

\section{AUTHOR CONTRIBUTIONS}

L-MZ, Y-MZ, and W-HL designed and supervised this study. J-MG, LL, R-TC, J-JZ contributed to data acquisition, and Y-FK analyzed the data. L-MZ, Y-MZ, and W-HL wrote and revised the manuscript. All authors contributed to the article and approved the submitted version.

\section{FUNDING}

This study was funded by the National Natural Science Foundation of China, Grant/Award Numbers: 82001784,

\section{REFERENCES}

1. Ashburner J. A Fast Diffeomorphic Image Registration Algorithm. Neuroimage (2007) 38(1):95-113. doi: 10.1016/j.neuroimage.2007.07.007

2. Cavanna AE, Trimble MR. The Precuneus: A Review of Its Functional Anatomy and Behavioural Correlates. Brain: J Neurol (2006) 129(Pt 3):564-83. doi: 10.1093/brain/awl004

3. Chao-Gan Y, Yu-Feng Z. DPARSF: A MATLAB Toolbox for "Pipeline" Data Analysis of Resting-State fMRI. Front Syst Neurosci (2010) 4:13. doi: 10.3389/ fnsys.2010.00013

4. Chen Q, Lv X, Zhang S, Lin J, Song J, Cao B, et al. Altered Properties of Brain White Matter Structural Networks in Patients With Nasopharyngeal Carcinoma After Radiotherapy. Brain Imaging Behav (2020) 14(6):2745-61. doi: 10.1007/s11682-019-00224-2

5. Chen Y-P, Chan ATC, Le Q-T, Blanchard P, Sun Y, Ma J. Nasopharyngeal Carcinoma. Lancet (London England) (2019) 394(10192):64-80. doi: 10.1016/ S0140-6736(19)30956-0

6. Coderre JA, Morris GM, Micca PL, Hopewell JW, Verhagen I, Kleiboer BJ, et al. Late Effects of Radiation on the Central Nervous System: Role of Vascular Endothelial Damage and Glial Stem Cell Survival. Radiat Res (2006) 166(3):495-503. doi: 10.1667/RR3597.1

7. Cohen AD, Tomasi D, Shokri-Kojori E, Nencka AS, Wang Y. Functional Connectivity Density Mapping: Comparing Multiband and Conventional EPI Protocols. Brain Imaging Behav (2018) 12(3):848-59. doi: 10.1007/s11682017-9742-7

8. Ding Z, Zhang H, Lv X-F, Xie F, Liu L, Qiu S, et al. Radiation-Induced Brain Structural and Functional Abnormalities in Presymptomatic Phase and Outcome Prediction. Hum Brain Mapp (2018) 39(1):407-27. doi: 10.1002/ hbm. 23852

9. Esassolak M, Karagöz U, Yalman D, Köse S, Anacak Y, Haydaroğlu A. Evaluation of the Effects of Radiotherapy to the Chiasm and Optic Nerve by Visual Psychophysical and Electrophysiologic Tests in Nasopharyngeal Carcinoma. Int J Radiat Oncol Biol Phys (2004) 58(4):1141-6. doi: 10.1016/ j.ijrobp.2003.08.014

10. Greicius MD, Krasnow B, Reiss AL, Menon V. Functional Connectivity in the Resting Brain: A Network Analysis of the Default Mode Hypothesis. Proc Natl Acad Sci (2003) 100(1):253-8. doi: 10.1073/pnas.0135058100

11. Jang SH, Kim SH, Seo JP. Spinothalamic Tract Injury Due to Primary Brainstem Injury: A Case Report. Am J Phys Med Rehabil (2016) 95(3):e4243. doi: $10.1097 / \mathrm{phm} .0000000000000414$
91959117, 82071894; The Youth Science Foundation of Xiangya Hospital, Grant/Award Number: 2019Q16; The Natural Science Foundation (General Project) of Hunan Province, Grant/Award Number: 2018JJ2271.

\section{ACKNOWLEDGMENTS}

The authors were all grateful to all subjects who joined this study. We thank Bronwen Gardner, PhD, from Liwen Bianji (Edanz) (www.liwenbianji.cn/ac) for editing the English text of this manuscript.

\section{SUPPLEMENTARY MATERIAL}

The Supplementary Material for this article can be found online at: https://www.frontiersin.org/articles/10.3389/fonc.2021. 687127/full\#supplementary-material

12. Jensen KB, Regenbogen C, Ohse MC, Frasnelli J, Freiherr J, Lundström JN. Brain Activations During Pain. Pain (2016) 157(6):1279-86. doi: 10.1097/ j.pain.0000000000000517

13. Kravitz DJ, Saleem KS, Baker CI, Ungerleider LG, Mishkin M. The Ventral Visual Pathway: An Expanded Neural Framework for the Processing of Object Quality. Trends Cogn Sci (2013) 17(1):26-49. doi: 10.1016/j.tics.2012.10.011

14. Lee AW, Law SC, Ng SH, Chan DK, Poon YF, Foo W, et al. Retrospective Analysis of Nasopharyngeal Carcinoma Treated During 1976-1985: Late Complications Following Megavoltage Irradiation. Br J Radiol (1992) 65 (778):918-28. doi: 10.1259/0007-1285-65-778-918

15. Lee AW, Ng SH, Ho JH, Tse VK, Poon YF, Tse CC, et al. Clinical Diagnosis of Late Temporal Lobe Necrosis Following Radiation Therapy for Nasopharyngeal Carcinoma. Cancer (1988) 61(8):1535-42. doi: 10.1002/ 1097-0142(19880415)61:8<1535::AID-CNCR2820610809>3.0.CO;2-E

16. Lee AW, Ng WT, Pan JJ, Chiang CL, Poh SS, Choi HC, et al. International Guideline on Dose Prioritization and Acceptance Criteria in Radiation Therapy Planning for Nasopharyngeal Carcinoma. Int J Radiat Oncol Biol Phys (2019) 105(3):567-80. doi: 10.1016/j.ijrobp.2019.06.2540

17. Lee CC, Ho CY. Post-Treatment Late Complications of Nasopharyngeal Carcinoma. Eur Arch Otorhinolaryngol (2012) 269(11):2401-9. doi: 10.1007/ s00405-011-1922-2

18. Leng X, Fang P, Lin H, Qin C, Tan X, Liang Y, et al. Application of a Machine Learning Method to Whole Brain White Matter Injury After Radiotherapy for Nasopharyngeal Carcinoma. Cancer Imaging: Off Publ Int Cancer Imaging Soc (2019) 19(1):19. doi: 10.1186/s40644-019-0203-y

19. Li W, Zhang J, Zhou C, Hou W, Hu J, Feng H, et al. Abnormal Functional Connectivity Density in Amyotrophic Lateral Sclerosis. Front Aging Neurosci (2018) 10:215. doi: 10.3389/fnagi.2018.00215

20. Liang YF, Kong B, Xiang WY, Ruan JB, Wang LM, Chen C, et al. Nasopharyngeal Adenoid Cystic Carcinoma: A Case Report and Review of the Literature. Int J Clin Exp Pathol (2014) 7(7):4516-8. doi: 10.1016/ j.amsu.2018.04.004

21. Lin J, Lv X, Niu M, Liu L, Chen J, Xie F, et al. Radiation-Induced Abnormal Cortical Thickness in Patients With Nasopharyngeal Carcinoma After Radiotherapy. NeuroImage Clin (2017) 14:610-21. doi: 10.1016/j.nicl. 2017.02.025

22. Liu Y, Yu C, Zhang X, Liu J, Duan Y, Alexander-Bloch AF, et al. Impaired Long Distance Functional Connectivity and Weighted Network Architecture in Alzheimer's Disease. Cereb Cortex (New York N.Y. 1991) (2014) 24 (6):1422-35. doi: 10.1093/cercor/bhs410 
23. Ma Q, Wu D, Zeng LL, Shen H, Hu D, Qiu S. Radiation-Induced Functional Connectivity Alterations in Nasopharyngeal Carcinoma Patients With Radiotherapy. Med (Baltimore) (2016) 95(29):e4275. doi: 10.1097/md. 0000000000004275

24. Mak LE, Minuzzi L, Macqueen G, Hall G, Kennedy SH, Milev R. The Default Mode Network in Healthy Individuals: A Systematic Review and MetaAnalysis. Brain Connectivity (2017) 7(1):25-33. doi: 10.1089/brain.2016.0438

25. Shen Q, Lin F, Rong X, Yang W, Li Y, Cai Z, et al. Temporal Cerebral Microbleeds Are Associated With Radiation Necrosis and Cognitive Dysfunction in Patients Treated for Nasopharyngeal Carcinoma. Int J Radiat Oncol Biol Phys (2016) 94(5):1113-20. doi: 10.1016/j.ijrobp. 2015.11.037

26. Qiu Y, Guo Z, Han L, Yang Y, Li J, Liu S, et al. Network-Level Dysconnectivity in Patients With Nasopharyngeal Carcinoma (NPC) Early Post-Radiotherapy: Longitudinal Resting State fMRI Study. Brain Imaging Behav (2018) 12 (5):1279-89. doi: 10.1007/s11682-017-9801-0

27. Schrouff J, Rosa MJ, Rondina JM, Marquand AF, Chu C, Ashburner J, et al. PRoNTo: Pattern Recognition for Neuroimaging Toolbox. Neuroinformatics (2013) 11(3):319-37. doi: 10.1007/s12021-013-9178-1

28. Shi L, Du F-L, Sun Z-W, Zhang L, Chen Y-Y, Xie T-M, et al. RadiationInduced Gray Matter Atrophy in Patients With Nasopharyngeal Carcinoma After Intensity Modulated Radiotherapy: A MRI Magnetic Resonance Imaging Voxel-Based Morphometry Study. Quantitative Imaging Med Surg (2018) 8(9):902-9. doi: 10.21037/qims.2018.10.09

29. Tomasi D, Volkow ND. Functional Connectivity Density Mapping. Proc Natl Acad Sci USA (2010) 107(21):9885-90. doi: 10.1073/pnas.1001 414107

30. Tomasi D, Volkow ND. Functional Connectivity Hubs in the Human Brain. NeuroImage (2011) 57(3):908-17. doi: 10.1016/j.neuroimage. 2011.05.024

31. Tomasi D, Volkow ND. Abnormal Functional Connectivity in Children With Attention-Deficit/Hyperactivity Disorder. Biol Psychiatry (2012) 71(5):44350. doi: 10.1016/j.biopsych.2011.11.003

32. Zhang Y-M, Chen M-N, Yi X-P, Li L, Gao J-M, Zhang J-L, et al. Cortical Surface Area Rather Than Cortical Thickness Potentially Differentiates Radiation Encephalopathy at Early Stage in Patients With Nasopharyngeal Carcinoma. Front Neurosci (2018) 12:599. doi: 10.3389/fnins.2018.00599

33. Torre LA, Bray F, Siegel RL, Ferlay J, Lortet-Tieulent J, Jemal A. Global cancer statistics, 2012. CA: Cancer J Clin (2015) 65(2):87-108. doi: 10.3322/ caac. 21262
34. Van Dijk KRA, Sabuncu MR, Buckner RL. The Influence of Head Motion on Intrinsic Functional Connectivity MRI. NeuroImage (2012) 59(1):431-8. doi: 10.1016/j.neuroimage.2011.07.044

35. Wang Y-XJ, King AD, Zhou H, Leung S-F, Abrigo J, Chan Y-L, et al. Evolution of Radiation-Induced Brain Injury: MR Imaging-Based Study. Radiology (2010) 254(1):210-8. doi: 10.1148/radiol.09090428

36. Xia M, Wang J, He Y. BrainNet Viewer: A Network Visualization Tool for Human Brain Connectomics. PloS One (2013) 8(7):e68910. doi: 10.1371/ journal.pone.0068910

37. Yan CG, Wang XD, Zuo XN, Zang YF. DPABI: Data Processing \& Analysis for (Resting-State) Brain Imaging. Neuroinformatics (2016) 14(3):339-51. doi: 10.1007/s12021-016-9299-4

38. Zhang B, Lian Z, Zhong L, Zhang X, Dong Y, Chen Q, et al. Machine-Learning Based MRI Radiomics Models for Early Detection of Radiation-Induced Brain Injury in Nasopharyngeal Carcinoma. BMC Cancer (2020) 20(1):502. doi: 10.1186/s12885-020-06957-4

39. Zhang B, Mo Z, Du W, Wang Y, Liu L, Wei Y. Intensity-Modulated Radiation Therapy Versus 2D-RT or 3D-CRT for the Treatment of Nasopharyngeal Carcinoma: A Systematic Review and Meta-Analysis. Oral Oncol (2015) 51 (11):1041-6. doi: 10.1016/j.oraloncology.2015.08.005

40. Zhang Y-M, Gao J-M, Zhou H, Li L, Liu L-Z, Han Z-D, et al. Pre-Symptomatic Local Brain Activity and Functional Connectivity Alterations in Nasopharyngeal Carcinoma Patients Who Developed Radiation Encephalopathy Following Radiotherapy. Brain Imaging Behav (2019) 145 (5):1964-78. doi: 10.1007/s11682-019-00145-0

41. Zuo X-N, Ehmke R, Mennes M, Imperati D, Castellanos FX, Sporns O, et al. Network Centrality in the Human Functional Connectome. Cereb Cortex (New York N.Y.) (2012) 22(8):1862-75. doi: 10.1093/cercor/bhr269

Conflict of Interest: The authors declare that the research was conducted in the absence of any commercial or financial relationships that could be construed as a potential conflict of interest.

Copyright (C) 2021 Zhao, Kang, Gao, Li, Chen, Zeng, Zhang and Liao. This is an openaccess article distributed under the terms of the Creative Commons Attribution License (CC BY). The use, distribution or reproduction in other forums is permitted, provided the original author(s) and the copyright owner(s) are credited and that the original publication in this journal is cited, in accordance with accepted academic practice. No use, distribution or reproduction is permitted which does not comply with these terms. 